\title{
Biologic Disease-Modifying Antirheumatic Drug
}

National Cancer Institute

\section{Source}

National Cancer Institute. Biologic Disease-Modifying Antirheumatic Drug. NCI

Thesaurus. Code C121334.

A biologic used to alter the progression of autoimmune disease. 